\title{
NONLINEAR PULSATIONAL BEHAVIOUR OF BW VULPECULAE
}

\author{
P. MOSKALIK ${ }^{1}$ and J.R. BUCHLER ${ }^{2}$ \\ ${ }^{1}$ N. Copernicus Astronomical Center, Bartycka 18, 00-716 Warsaw, Poland \\ ${ }^{2}$ Department of Physics, University of Florida, Ganesville, FL32611, USA
}

BW Vulpeculae is a large amplitude $\beta$ Cephei-type star, pulsating with a single period of 0.20104 day. Its nonlinear modeling was first attempted by Pesnell \& Cox (1980). Because the true instability mechanism was not known then, the pulsation amplitude was imposed in that study in an arbitrary fashion.

The driving of $\beta$ Cephei-type pulsations is now well understood (e.g. Dziembowski \& Pamyatnykh 1993) and the hydrodynamical modeling can be done today in a fully self-consistent way. For this purpose we study 120 zone envelope models extending down to $T=3 \times 10^{7} \mathrm{~K}$. We use the OPAL opacities (Iglesias, Rogers \& Wilson 1992) for the metallicity of $Z=0.02$. The models are constructed as to lie on the evolutionary tracks of Dziembowski \& Pamyatnykh (1993) and are chosen to match BW Vul's pulsation period and $\log T_{\text {eff }}\left(\log T_{\text {eff }}=4.362-4.366\right.$; Heynderickx 1991). The radial pulsations of the models are then studied with the relaxation hydrocode (Stellingwerf 1974), which yields strictly periodic full amplitude solutions (limit cycles).

In most of the models of this survey both the fundamental mode and the first radial overtone are linearly unstable. However, the overtone limit cycle has in all studied cases too small an amplitude to square with observations. In addition, this limit cycle is always unstable towards the excitation of the fundamental mode. Therefore, BW Vul almost certainly cannot be a first overtone pulsator.

For the fundamental mode, the hydrodynamical calculations reproduce the observed light and radial velocity curves reasonably well (Fig. 1). In particular, the large amplitudes of both curves are correctly modeled, although the lightcurve amplitude is somewhat too large. The jump and the following standstill in the velocity curve, which are the most outstanding observed features, are also reproduced. The standstill is caused by an emerging shock wave which originates at the bottom of the $\mathrm{He}^{+}$ionization zone (Fig. 2). The strong compression ocurring in the shock (14-fold increase of $\rho$ in less then $0.02 P$ ) results in an almost five-fold increase in the Rosseland-mean opacity. The sudden jump in the optical depth contributes to the formation of the apparent discotinuity in the observed radial velocities.

We find that the shock wave is stronger for more centrally condensed models in the secondary contraction phase. The resultant standstill is lo- 

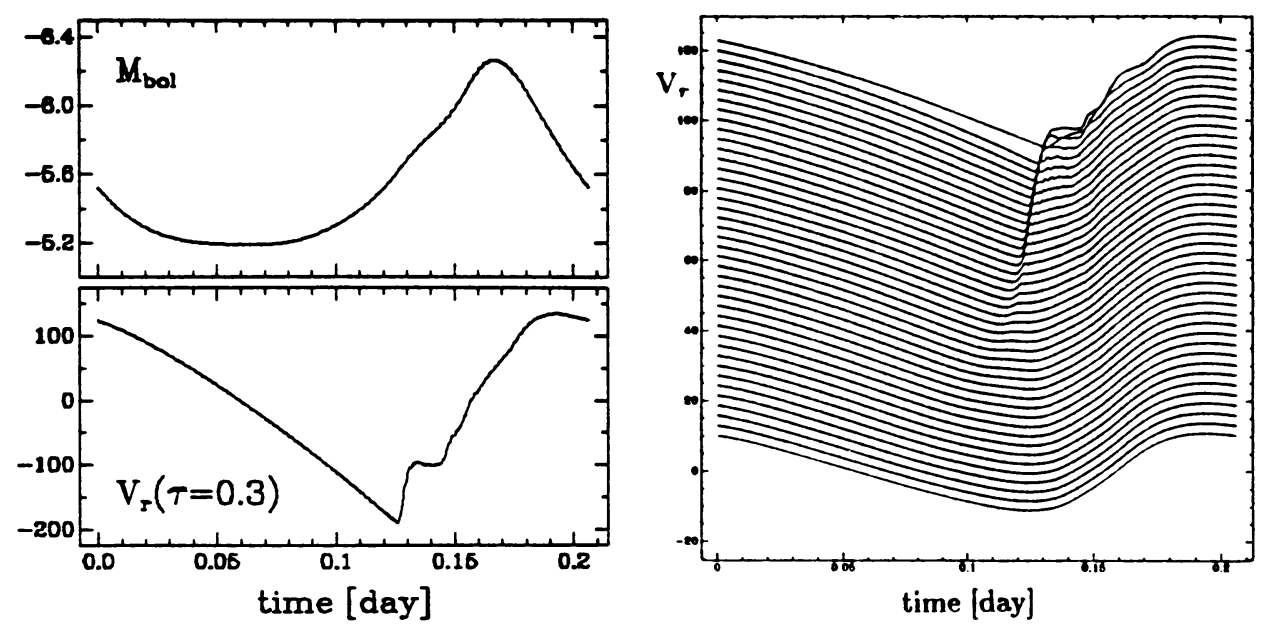

Fig. 1. Variations of bolometric magnitude (top) and radial velocities at constant $\tau=0.3$ (bottom) for a BW Vul model of $M=11 M_{\odot}, \log \left(L / L_{\odot}\right)=4.1457$ and $\log T_{\text {eff }}=4.3658$. The model is in the Main Sequence expansion phase and pulsates in the fundamental mode with $P=0.2068$ day. The velocity amplitude is $234 \mathrm{~km} / \mathrm{s}$ (after correcting with the factor of $17 / 24$ ) and is close to the observed value of $210 \mathrm{~km} / \mathrm{s}$ (Furenlid et al. 1987). The standstill in the velocity curve is due to an emerging shock wave. The theoretical lightcurve amplitude is $1.06 \mathrm{mag}$. This is close to the far-UV amplitude (1.16 mag), but larger than the estimated bolometric amplitude of $0.75 \mathrm{mag}$ (Barry et al. 1984). No standstill appears in the lightcurve.

Fig. 2. Radial velocities versus time for the outer zones of the BW Vul model of Fig. 1 . The shock wave propagating outwards originates at the bottom of the $\mathrm{He}^{+}$ionization zone.

cated in this case nearly exactly in the middle of the raising branch of the velocity curve. Such behaviour is in a better agreement with what is actually observed. This result suggests, that BW Vul is most likely evolved beyond the Main Sequence expansion phase.

The results presented here are preliminary. The full discussion of our modelling will be presented elswhere.

\section{References}

Barry, D.C., Holberg, J.B., Forrester, W.T., Polidan, R.S. and Furenlid, I.: 1984, ApJ. 281, 766.

Dziembowski, W.A. and Pamyatnykh, A.A.: 1993, MNRAS 262, 204

Furenlid, I., Young, A., Meylan, T., Haag, C. and Crinklaw, G.: 1987, ApJ. 319, 264

Heynderickx, D.: 1991, Ph. D. Thesis, Katholieke Universiteit Leuven.

Iglesias, C.A., Rogers, F.J. and Wilson, B.G.: 1992, ApJ. 397, 717

Pesnell, W.D. and Cox, A.N.: 1980, Space Sci. Rev. 27, 337

Stellingwerf, R.F.: 1974, ApJ. 192, 139 